\title{
A Few Considerations on Structural and Logical Composition in Specification Theories
}

\author{
Axel Legay \\ INRIA Rennes, France \\ axel.legay@irisa.fr
}

\author{
Andrzej Wąsowski \\ IT University of Copenhagen, Denmark \\ wasowski@itu.dk
}

\begin{abstract}
Over the last 20 years a large number of automata-based specification theories have been proposed for modeling of discrete, real-time and probabilistic systems. We have observed a lot of shared algebraic structure between these formalisms. In this short abstract, we collect results of our work in progress on describing and systematizing the algebraic assumptions in specification theories.
\end{abstract}

\section{Introduction}

Specification formalisms commonly support two main ways of combining specifications: conjunction and parallel composition. The former, conjunction, is more common in specification logics (such as temporal logics [4]). It focuses on combining distinct views on the same system, or a component. The latter, parallel composition, more often discussed in process algebraic approaches [5], talks about structurally composing two or more communicating systems.

Recently a few specification theories have been developed that support both parallel composition and conjunction (for example [7, 6]). Interestingly, placing both parallel composition and conjunction in the same theoretical framework raises a lot of natural questions. When presenting the results of [7] and [6], we have received a wide range of opinions about the two operators, ranging from composition and conjunction are radically different, through they are easy to confuse, and hard to distinguish to extreme ones such as conjunction and parallel composition are essentially the same. A more diligent insight into the properties of the two operators shows that each of the opinions is in a way justified. For example conjunction satisfies the most essential axioms of parallel composition in some cases, and both operators are computed as pruning results of a certain kind of product.

In this note we collect most important observations about similarities, relations and differences between conjunction and parallel composition in specification theories, as we have experienced in our prior work. First, we show that parallel composition refines conjunction for a broad class of single-player specification theories. Under the rather general assumptions that conjunction is the greatest-lower bound with respect to refinement, and the refinement is a precongruence for parallel composition, we show that existence of a certain kind of universal specification suffices for parallel composition to be always a stronger operator than conjunction (stronger as defined by the refinement relation). This fact is easily observed using basic properties of the order theory. It is not necessary to assume any specific properties about the specification language. An interesting side-effect is that parallel composition behaves much like conjunction under these assumptions. For example composing more systems to an existing assembly strengthens the specification (in the sense that it decreases the set of models).

These observations apply out of the box to theories of Constraint Markov Chains [6] and modal transition systems (scattered across multiple papers [9, 10, 11, 8]). It would also apply to a natural theory that one could build with finite automata and language inclusion as refinement. 
The existence of a universal specification requires an implicit closed-world assumption of a kind, which tends to hold in specification theories with a single-player semantics, like the above listed. Such theories do not distinguish between choices made by the component, and choices set by the environment in which it operates. In contrast one cannot always assume existence of a single largest specification when modeling under the open-world assumption. Consequently the above relation between parallel composition and conjunction does not hold for two-player specification theories such as Interface Automata [1], Timed Interfaces [3] or Timed I/O Automata [7]. For these models we can spot an interesting duality between the two operators.

Type theories, and older specification theories used in verification typically focus on universal correctness using a pessimistic composition, i.e. two systems are compatible, if under no circumstances their execution can lead to an error. In [1] Alfaro and Henzinger propose an optimistic composition operator, which stipulates that two components are compatible as long as there exists a context in which they will not fail. In [7, 2] this duality between optimistic-pessimistic is investigated further. In [7] we observe that the pessimistic composition is obtained by synthesizing a winning strategy for the player representing the system, while the optimistic composition is obtained by synthesizing a winning strategy for the player representing the environment.

Furthermore, the two compositions suit well two different use cases. When an implementer receives a contract specification for the component, the environment assumptions are given, while some choices in the implementation still remain under his control. Thus if constructing specifications for implementers the pessimistic composition is more suitable. In practice this means that conjunction should be computed as the strategy for the component, since conjunction is a natural way of constructing a contract.

Dually, the user of the component typically cannot influence the component itself, but can change the details of how it is used. This is why the optimistic composition is more suitable when synthesizing implementations for the users of black-box components. In this scenario parallel composition is the natural composition operator, and since the usage scenario is the only element under control, the composition is computed as the winning strategy for the environment player.

\section{A Single Player Setting}

In the following let $\mathbf{S}$ be a universe of specifications.

A1 Let $(\leq) \subseteq(\mathbf{S} \times \mathbf{S})$ be a binary relation on this universe. We call it a refinement. Our refinement induces an equivalence on specifications: $A \equiv B$ iff $A \leq B$ and $B \leq A$.

A2 We assume that $\leq$ is a pre-order (reflexive and transitive).

A3 We postulate existence of a universal specification $\mathrm{U}$, such that $A \leq \mathrm{U}$ for all $A \in \mathbf{S}$.

A4 Let conjunction be a binary operator: $\wedge: \mathbf{S} \times \mathbf{S} \rightarrow \mathbf{S}$.

A5 Conjunction is total : $A \wedge B$ is defined for all $A, B \in \mathbf{S}$.

A6 Conjunction is commutative: $A \wedge B \leq B \wedge A$ for all $A, B \in \mathbf{S}$.

Conjunction is the greatest lower bound with respect to $\leq$ :

A7 $A \wedge B \leq A$ and $A \wedge B \leq B$ for all $A, B \in \mathbf{S}$.

A8 If $C \leq A$ and $C \leq B$ then $C \leq A \wedge B$

A9 Another operator (|) : $\mathbf{S} \times \mathbf{S} \rightarrow \mathbf{S}$ is called parallel composition. 
A10 Total: $A \mid B$ is defined for all $A, B \in \mathbf{S} .^{1}$

A11 Commutative: $A|B \leq B| A$ for all $A, B$.

A12 Refinement is a precongruence for the contexts defined by parallel composition, so:

$$
A \leq B \text { implies that } A|C \leq B| C
$$

We additionally require that $\mathrm{U}$ is a unit of parallel composition:

A13 $A \mid \mathrm{U} \leq A$ for all specifications $A$

At first the above may appear a strong requirement, but in fact this is very natural, and holds in many specification theories. For example if ' $\mid$ ' is a product of automata then the product of $A$ with a universal automaton $\mathrm{U}$ gives $A$ again. In a more complicated scenario of CCS-like synchronizations where alphabets of $A$ and $U$ may differ, the refinement enforces alphabet equalization, which normally is defined by $A \mid \mathrm{U}$ and automatically gives the above.

Theorem 1. $A \mid B \leq A \wedge B$

Proof. Note that $A \leq \mathrm{U}$ so by precongruence $A|B \leq \mathrm{U}| B \leq B$ (the latter by assumptions A11 and A13). We conclude similarly that $A \mid B \leq A$ and since conjunction is the greatest lower bound $A \mid B \leq A \wedge B$.

As an example, observe that assumptions of Thm. 1 hold for a theory build around Constraint Markov Chains [6], Modal Specifications (not fully built in any paper, but results are scattered across more papers $[9,10,11,8])$, or just finite automata with language inclusion as refinement.

The condition $\mathrm{A} 13$, that $A \mid \mathrm{U} \leq A$, is in fact the sufficient and necessary condition for Thm. 1 to hold, in the following sense.

Theorem 2. If the universal element exists, and the refinement is a precongruence, and conjunction is the greatest lower bound, but $A \mid \mathrm{U} \not \leq A$ then not necessarily $A \mid B \leq A \wedge B$. hold.

This is easy to show by contradiction. Assume $A \mid \mathrm{U} \leq A \wedge \mathrm{U}$ but $A \mid \mathrm{U} \not \leq A$. Then $A \wedge \mathrm{U} \leq A$, so $A \mid \mathrm{U} \leq A \wedge \mathrm{U} \leq A$ so $A \mid \mathrm{U} \leq A$ - contradiction. Since the other conditions are typically required of any well structured specification theory, we conclude that if the universal specification exists then $A \mid \mathrm{U} \leq A$ is the sufficient and necessary condition for the parallel composition being always stronger than conjunction.

There is an interesting open question left by the above observations. We do know that the parallel composition, under certain conditions, is stronger than conjunction. However conjunction fulfills all the axioms of parallel composition in this setting (precongruence in particular). It would be interesting to explain more precisely what is it that makes parallel composition stronger than conjunction, and what is the weakest parallel composition that is interesting.

A14 Define the quotient of conjunction $B \backslash^{\wedge} A$ to be a greatest (with respect to $\leq$ ) specification $X$ such that $A \wedge X \leq B$.

A15 Define the quotient of parallel composition $B \backslash^{\mid} A$ to be a greatest (with respect to $\leq$ ) specification $X$ such that $A \mid X \leq B$.

For the moment ignore the problem that a unique quotient (up to the equivalence induced by $\leq$ ) may not exist. We will come back to this later.

\footnotetext{
${ }^{1}$ Totality of parallel composition is not strictly needed, but added to this note to simplify assumptions of further theorems and properties - in principle it is enough to always assume existance of parallel compositions in question.
} 
Theorem 3. Consider arbitrary specifications $A$ and $B$. If $B \backslash^{\wedge} A$ and $B \backslash^{\backslash} A$ are both defined (uniquely up to equivalence induced by $\leq$ ) then $B \backslash^{\wedge} A \leq B \backslash^{\wedge} A$.

Proof. Observe that $A \mid\left(B \backslash^{\wedge} A\right) \leq A \wedge\left(B \backslash^{\wedge} A\right) \leq B$. So by uniqueness and maximality of $B \backslash^{\backslash} A$ we must have that $B \backslash^{\wedge} A \leq B \backslash^{\backslash} A$ (as the former also fulfils the condition of the latter).

It is more interesting to ask the question of quotient's existance.

A16 Assume that conjunction has a null element, so: $A \wedge \emptyset \leq \emptyset$.

A17 Assume that $\vee$ is a total least upperbound operator in specifications (a disjunction):

A18 $A \leq C$ and $B \leq C$ implies that $A \vee B \leq C$

A19 $A \leq A \vee B$ and $B \leq A \vee B$.

A20 Assume that conjunction distributes over disjunction, so: $\left(A \wedge X_{1}\right) \vee\left(A \wedge X_{2}\right) \leq B$ implies $A \wedge\left(X_{1} \vee\right.$ $\left.X_{2}\right) \leq B$.

Theorem 4. In specification theories, where the conjunction distributes over disjunction (in the sense of the above) we have that quotient always exists for conjunction (so $B \backslash^{\wedge} A$ is uniquely defined up to equivalence), if disjunction (lub) is defined for arbitrary (also infinite) sets.

Proof. First observe that $\emptyset$ is always a good quotient candidate.

If there is more than one quotient candidate then a least upper bound of all of them also fulfils the definition. So we take the quotient to be the least upper bound of all those that fulfill the definition.

We have been unable to find generic conditions for existence of quotient for parallel composition.

We shall now investigate associativity of conjunction and parallel composition. Order theory tells us that conjunction is associative:

Theorem 5. $(A \wedge B) \wedge C \leq A \wedge(B \wedge C)$

Proof. By A7: $(A \wedge B) \wedge C \leq C$

By A7 again: $(A \wedge B) \wedge C \leq A \wedge B \leq A$

Similarly: $(A \wedge B) \wedge C \leq B$

By A8: $(A \wedge B) \wedge C \leq(B \wedge C)$

By A8 again: $(A \wedge B) \wedge C \leq A \wedge(B \wedge C)$

To investigate associativity of parallel composition we additionally assume that parallel composition is idempotent:

A21 For all specifications $A \in \mathbf{S}$ have $A \leq A \mid A$.

Note that the opposite $A \mid A \leq A$ already follows from Theorem 1 and idempotence of conjunction. Now we obtain the following:

Theorem 6. For all specifications $A, B$, and $C$ we have $(A \mid B)|C \leq A|(B \mid C)$.

Proof. $B|C \geq(A \mid B)| C$ by Theorem 1, A7 and precongruence.

$A|(B \mid C) \geq A|[(A \mid B) \mid C] \geq(A \mid B)|[(A \mid B) \mid C] \geq[(A \mid B) \mid C]|[(A \mid B) \mid C] \geq(A \mid B) \mid C$. The last step is by idempotence of $\left.{ }^{\prime}\right|^{\prime}$. 
Observe that the above theorem, precongruence and commutativity of parallel composition also implies that $A|(B \mid C) \leq(A \mid B)| C$, which completes the expected property of associativity:

$$
A|(B \mid C) \leq(C \mid B)| A \leq C|(B \mid A) \leq(A \mid B)| C
$$

\section{In a Two-Player Game Setting}

It turns out that the assumptions of the previous section do not hold for a particular class of theories-those based on two player games. For example there does not exist a universal interface automaton [1] while a universal timed specification [7], if any was proposed, would not fulfill $A \mid \mathrm{U} \leq A$. Let us explain below, why this is the case.

Specifications with a game semantics, separate actions into controllable by the component (outputs) and by the environment (inputs). In such settings the parallel composition is only possible for two components, for which the controllable parts of the alphabet do not overlap-otherwise we have a control conflict. This is not required for conjunction, so conjunction exists more often than parallel composition in such theories (and assumption A10 is violated). In particular this means that $A \mid \mathrm{U}$ would typically need to have both an alphabet identical to $A$ and different than $A$ (for example in interface automata).

Moreover an optimistic parallel composition [1] is specified as a maximum wining strategy for the input player (the player corresponding to the context of the composition) in a safety game. Dually conjunction is constructed as a maximum winning strategy for the output player (the player representing the conjunction component itself) in a safety game. This duality expresses very well the difference between the two operators. The former is concerned with correct use of the result-and use is the domain of the context per se. The latter is concerned with the realization of two specifications-this clearly should be resolved within the component, and not within the environment.

We have fully developed this principle, when working on a real time specification theory [7].

\section{Concluding Remarks and Future Work}

The remarks placed in this short paper are clearly very preliminary. It is our intention to investigate more in depth algebraic structures underlying specification theories, and use this study to better survey existing theories, and to systematize the design of the new ones.

\section{References}

[1] L. de Alfaro \& T. A. Henzinger (2001): Interface automata. In: FSE, ACM Press, pp. 109-120.

[2] Luca de Alfaro \& Thomas A. Henzinger (2001): Interface Theories for Component-Based Design. In: Thomas A. Henzinger \& Christoph M. Kirsch, editors: EMSOFT, Lecture Notes in Computer Science 2211, Springer, pp. 148-165.

[3] Luca de Alfaro, Thomas A. Henzinger \& Mariëlle Stoelinga (2002): Timed Interfaces. In: Alberto L. Sangiovanni-Vincentelli \& Joseph Sifakis, editors: EMSOFT, Lecture Notes in Computer Science 2491, Springer, pp. 108-122.

[4] Christel Baier \& Joost-Pieter Katoen (2008): Principles of Model Checking. The MIT Press.

[5] Jan A. Bergstra, Alban Ponse \& Scott A. Smolka, editors (2001): Elsevier.

[6] Benoit Caillaud, Benoît Delahaye, Kim G. Larsen, Axel Legay, Mikkel L. Pedersen \& Andrzej Wasowski (2010): Compositional Design Methodology with Constraint Markov Chains. In: QEST'10. To appear. 
[7] Alexandre David, Kim G. Larsen, Axel Legay, Ulrik Nyman \& Andrzej Wasowski (2010): Timed I/O automata: a complete specification theory for real-time systems. In: Karl Henrik Johansson \& Wang Yi, editors: HSCC, ACM ACM, pp. 91-100.

[8] G. Goessler \& J.-B. Raclet (2009): Modal Contracts for Component-based Design. In: Proc. of the 7th IEEE International Conference on Softwar e Engineering and Formal Methods (SEFM'09), IEEE Computer Society Press, Hanoi, Vietnam. To appear.

[9] K. G. Larsen (1989): Modal Specifications. In: AVMS, LNCS 407, Springer, pp. 232-246.

[10] Kim Guldstrand Larsen, Ulrik Nyman \& Andrzej Wąsowski (2007): Modal I/O Automata for Interface and Product Line Theories. In: ESOP, LNCS, Springer, pp. 64-79.

[11] J-B. Raclet, E. Badouel, A. Benveniste, B. Caillaud, A. Legay \& R. Passerone (2009): Modal Interfaces: Unifying Interface Automata and Modal Specifications. In: EMSOFT. 